\title{
Errata: Nonequilibrium Discontinuous Phase Transitions in a Fast Ionic Conductor Model: Coexistence and Spinodal Lines ${ }^{1}$
}

\author{
J. Marro ${ }^{2,3}$ and J. L. Vallés ${ }^{2,4}$ \\ Received December 17, 1987
}

In the first paragraph on page 123 the sentence that goes, "... where $N=L^{2}$ and $n_{i}=1$ (ion) or 0 (hole), is the occupation variable at each lattice site $i=1, \ldots, N$, and is allowed...," should read, "... where $N=L^{2}$ and $n_{i}=1$ (ion) or 0 (hole) is the occupation variable at each lattice site $i=1, \ldots, N$, is also allowed...."

In the last paragraph on page 126 the first sentence should start, "The fact that multistrip states are not real metastable states, however,...."

Also, in Table II on page 131 the left half of the values of the structure function correspond to a density $\rho=0.2$ and the right half to $\rho=0.35$.

\footnotetext{
${ }^{1}$ This paper appeared in J. Stat. Phys. 49:121 (1987).

${ }^{2}$ Departamento de Física Fonamental, Universitat de Barcelona, E-08028 Barcelona, Spain.

${ }^{3}$ Present address: Departamento de Física Aplicada, Facultad de Ciencias, Universidad de Granada, Granada, Spain.

${ }^{4}$ Present address: Courant Institute of Mathematical Sciences, New York University, 251 Mercer Street, New York, N.Y. 10012.
} 